\title{
Impedance simulations and measurements on the LHC collimators with embedded beam position monitors
}

\author{
N. Biancacci, F. Caspers, J. Kuczerowski, E. Métral, N. Mounet," and B. Salvant \\ CERN, CH-1211 Geneva 23, Switzerland
}

\author{
A. Mostacci \\ Rome University “La Sapienza," Piazzale Aldo Moro 5, 00185 Roma, Italy \\ O. Frasciello and M. Zobov \\ INFN-LNF, Via Enrico Fermi 40, 00044 Frascati (Roma), Italy
}

(Received 12 September 2016; published 12 January 2017)

\begin{abstract}
The LHC collimation system is a critical element for the safe operation of the LHC machine. The necessity of fast accurate positioning of the collimator's jaws, recently introduced the need to have button beam position monitors directly embedded in the jaws extremities of the LHC tertiary collimators and some secondary collimators. This addition led to a new design of these collimators including ferrites to damp higher order modes instead of rf fingers. In this work we will present the impedance bench measurements and simulations on a TCT (Transverse Tertiary Collimator) prototype including estimations for beam stability for the LHC.
\end{abstract}

DOI: 10.1103/PhysRevAccelBeams.20.011003

\section{INTRODUCTION}

The Large Hadron Collider (LHC) has a very sophisticated collimation system [1,2] used to protect the accelerator against regular and accidental beam losses. It is also used for beam cleaning purposes preventing halo particles deposition in the superconducting magnets, experimental detectors and other sensitive machine areas and hardware. The collimation system has a complex hierarchy composed of the primary (TCP), secondary (TCS), and tertiary (TCT) collimators and the injection protection collimators.

The LHC collimators have complex mechanical designs including movable jaws made of resistive materials, $\mathrm{rf}$ contacts, ferrite materials, cooling pipes etc. [3] to ensure stability under thermal and mechanical stress. Since the collimators are moved very close to the circulating beams they give the dominant contribution to the collider beam coupling impedance. Their broad-band impedance contributes mainly to the betatron tunes shifts with beam current and to several observed single bunch instabilities [4].

The need to control impedance was recognized already at the LHC design stage [5], many impedance related phenomena were observed during the LHC Run I [6] and are encountered in operating conditions [7]. The problem is expected to be even more severe for the High Luminosity

\footnotetext{
*Present address: Ecole Polytechnique Federale de Lausanne, Switzerland.

Published by the American Physical Society under the terms of the Creative Commons Attribution 3.0 License. Further distribution of this work must maintain attribution to the author(s) and the published article's title, journal citation, and DOI.
}

LHC upgrade [8,9] where one of the principal key ingredients for the luminosity increase is the beam current increase. That is why the impedance team has been paying much attention to a careful characterization of the beam coupling impedance of the LHC vacuum chamber components, and first of all the impedance of collimators, by using numerical simulations and experimental measurements.

During the first LHC long shutdown (LS1) 2 secondary collimators and 16 tertiary ones were replaced by new collimators with embedded beam position monitors (BPMs) [10]. The main purpose of using such collimators is to provide a better and faster collimator jaws alignment with respect to the stored beam orbit. In this way, a better cleaning efficiency is achieved relying on the accuracy of the gap size and jaw inclination control [11].

With respect to the existing collimators designs optimized from the impedance point of view, like the ones of high intensity accelerators as PEPII [12], LCLS [13], KEKB [14], SuperKEKB [15], DAFNE [16] and many others, the new LHC collimators have several distinct design features [17] that affect their coupling impedance: in addition to the two button BPMs, each jaw has now a double taper on either end. Such a design made the usage of rf contacts uneasy. At the request of the collimation project team and following some issues with rf contacts [18], the impedance team has recommended to leave the gap open, without the rf contacts, and to install special ferrite blocks to suppress parasitic higher order modes (HOMs) [19].

Considering the importance of the collimator beam impedance, a comprehensive study has been undertaken, both numerical and experimental, to quantify the impedance of the new collimators and to evaluate their eventual effect 
on the beam dynamics. In order to simulate the collimators as close as possible to their real designs [20] we used CAD drawings including all the mechanical details and real material properties (resistive walls, lossy ferrites) as an input for the GdfidL code [21]. A very fine mesh, typically of several billions mesh points, was required to reproduce the collimator detailed structure and to overcome arising numerical problems. In parallel to these CPU time consuming simulations we used also a simplified collimator model performing simulations with Computer Simulation Technology (CST) [22] in order to rapidly investigate parametric impedance dependencies (for example, on the collimators gaps, used ferrite materials etc.).

In 2014 an intensive campaign of impedance bench measurements was launched in order to characterize the impedance of the new collimators experimentally. A quite satisfactory agreement was found between the numerical predictions and the experimental data and observations. The obtained impedance has been implemented into the LHC impedance model for the following beam dynamics studies.

In this paper we briefly describe the design of the new collimators with embedded BPMs, discuss the results of numerical simulations and compare them with the available experimental data and, finally, evaluate the impact of the collimator impedance on the beam stability.

The paper is structured as follows. Section II describes the collimator design with emphasis on the features affecting the collimators impedance. Section III discusses the numerical simulations with GdfidL and CST. Section IV describes the experimental setup for the impedance measurements and summarizes the obtained experimental data comparing them with the simulations. Section V is dedicated to the beam stability studies using the refined LHC impedance model.

\section{DESIGN FEATURES}

The proposed TCT design, features the installation of embedded BPMs at the entrance and exit taper sections of

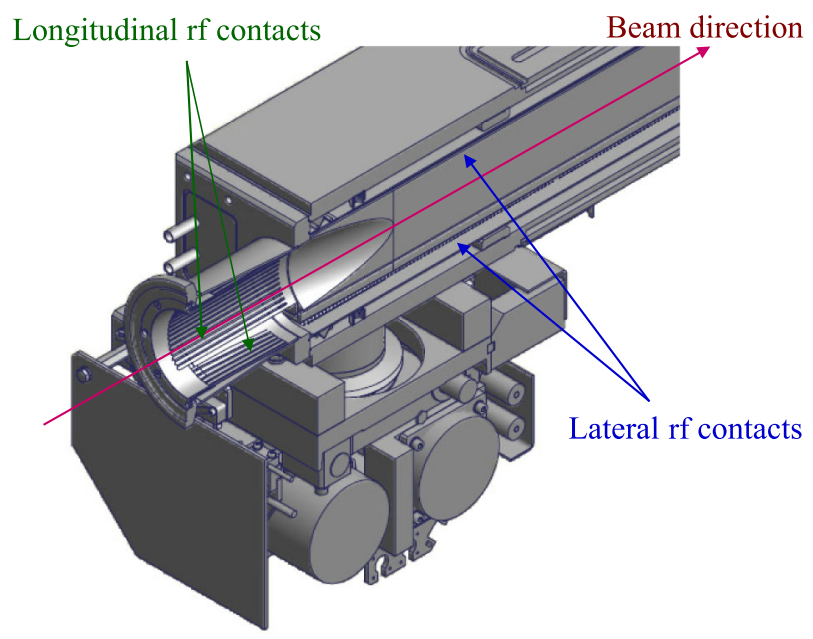

FIG. 1. TCT design with rf contacts.

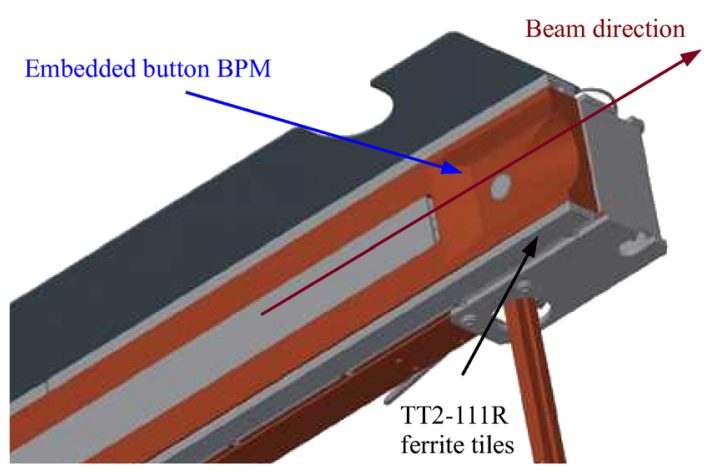

FIG. 2. TCT design with embedded BPMs and TT2-111R ferrite tiles.

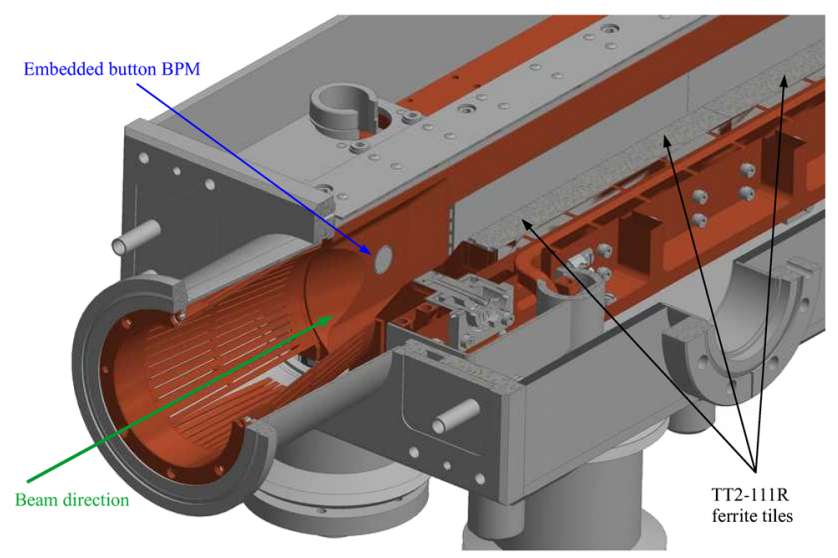

FIG. 3. LHC Run II TCS/TCT collimator with embedded BPM pickup buttons.

the collimator and the replacement of the rf contacts with TT2-111R ferrite tiles [23] as shown in Figs. 1 and 2. The presence of the BPMs requires an additional flat part in the taper to host the buttons, increasing the tapering section of the collimator (see Fig. 3) and therefore the contribution to the device impedance as evaluated in the next section. The choice of removing the contacts in favor of ferrite tiles is mainly dictated by possible dust production in operation and some observed impedance issues, especially with long rf fingers that would have been placed above the beam to screen the BPMs cables [18]. From the rf point of view, the removal of the rf contacts results in appearance of additional low frequency higher order modes. In turn, losses in the installed ferrite tiles help to reduce the shunt impedances and quality factors of these modes as it is discussed in the next section.

\section{NUMERICAL SIMULATIONS}

In order to simulate the collimators as close as possible to their real designs [20], we used CAD drawings including all the mechanical details as inputs for the parallelizable, UNIX-platform FDTD GdfidL code [21]. A very fine mesh, typically, of several billions mesh points, was required to reproduce the long and complicated structures, described in large .stl files, and to overcome arising numerical problems. 
In order to be sure that the code reproduces correctly the properties of lossy dispersive materials (resistive walls, ferrites) used in the collimators we have performed several dedicated numerical tests comparing the GdfidL simulations [24] with available analytical formulas, other numerical codes and semianalytical mode matching techniques [25]. The only way to afford such a complex computational task was to use the GdfidL dedicated cluster at CERN, "engpara," which has allowed us to study the wake fields and impedances for several types of collimators without using any model simplifications [26]. In such circumstances, GdfidL wake fields computation up to wake length of hundreds times the typical devices lengths (approximately $1.5 \mathrm{~m}$ ) took several days or two weeks at maximum.

\section{A. TCT GdfidL model}

The CAD drawing of the new collimator that was used in our simulations is shown in Fig. 3.

As discussed in the previous section, with respect to the previous LHC Run I TCS/TCT collimator design, several important changes were made: in order to accommodate the BPM button the jaw tapers were divided in two with a flat central part used for the BPM allocation; the lateral rf fingers were removed and HOM damping was entrusted to the TT2-111R ferrite blocks.

As the first step, we have evaluated how these changes affect the transverse broad band impedance. Namely this impedance is responsible for the single bunch transverse mode coupling instability (TMCI) and its imaginary part leads to an intensity dependent betatron tune shift. Since the effective imaginary broad-band impedance is proportional to the transverse loss (kick) factor (see, for example, [26]) we calculated the short range wake fields over the bunch length and compared the resulting kick factors for the old and new collimator designs for three different values of the half gap between the collimator jaws. As we see from Table I, the kick factors and, respectively, the effective imaginary impedance, is about $20 \%$ higher for the new collimators. This increase is mainly due to the steeper tapers in the collimator jaws. The impedance increase is nevertheless acceptable with respect to the LHC transverse impedance budget, where the geometric part of the collimator impedance contributes to $\simeq 20 \%$ on the total.

TABLE I. Geometric transverse kick factors due to the LHC Run I and Run II TCS/TCT geometries, calculated at different half gap values.

\begin{tabular}{lcc}
\hline \hline & w/ BPM & w/o BPM \\
\cline { 2 - 3 } Half gap $(\mathrm{mm})$ & $k_{T}\left(\frac{\mathrm{V}}{\mathrm{Cm}}\right)$ & $k_{T}\left(\frac{\mathrm{V}}{\mathrm{Cm}}\right)$ \\
\hline 1 & $3.921 \times 10^{14}$ & $3.340 \times 10^{14}$ \\
3 & $6.271 \times 10^{13}$ & $5.322 \times 10^{13}$ \\
5 & $2.457 \times 10^{13}$ & $2.124 \times 10^{13}$ \\
\hline \hline
\end{tabular}

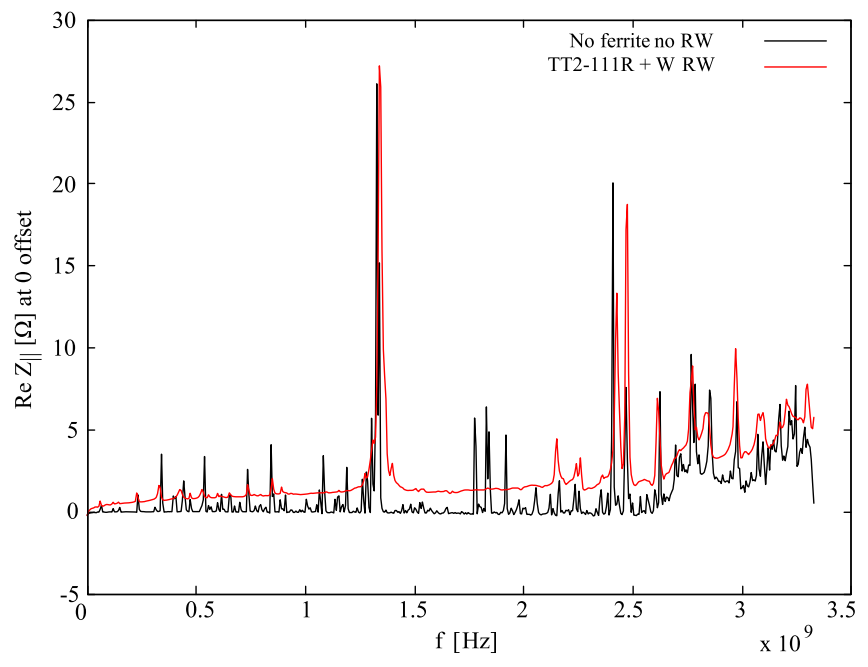

FIG. 4. Longitudinal impedance for the new collimator as computed by GdfidL, with (red curve) and without (black curve) TT2-111R ferrite and tungsten resistive wall (W). No offset is applied.

In order to study the narrow-band impedance behavior and the effectiveness of HOM damping with the ferrite blocks, we have performed detailed simulations of the real collimator structure tracing the wake fields over several hundred meters behind the bunch, until the wake fields completely decay. The resulting impedance was obtained by performing the Fourier transform of these wake potentials. The finite conductivity of Tungsten (W) and the frequency-dependent permeability of TT2-111R material were included in the simulation. The effect of the lossy dispersive materials on the longitudinal HOM damping in the new collimator is seen in Fig. 4: the black curve

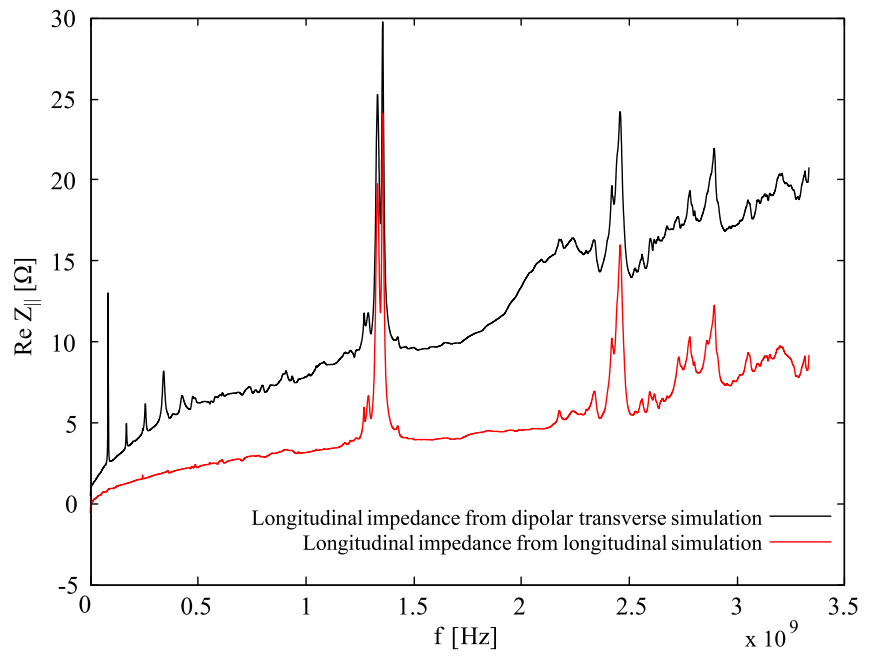

FIG. 5. Longitudinal impedances computed for a beam with zero transverse beam offset (red curve) and with a transverse beam offset as done in transverse dipolar impedance simulations (black curve). The computed low frequency HOMs correspond to transverse modes. 
represents the longitudinal narrow-band impedance of the collimator simulated as a whole perfectly conducting (PEC) structure (i.e., without any resistive and dispersive material), while the red one corresponds to the real collimators with $\mathrm{W}$ jaws and ferrite blocks.

We can see that the longitudinal higher order modes up to $1.2-1.3 \mathrm{GHz}$ are damped by the TT2-111R ferrite blocks and by the resistive wall contribution of the jaws. The low frequency mode damping is very important for the heating reduction of the collimators in the multibunch regime (keeping in mind that the nominal LHC bunches are $7.5 \mathrm{~cm}$ long). However, as we can see, the modes in the

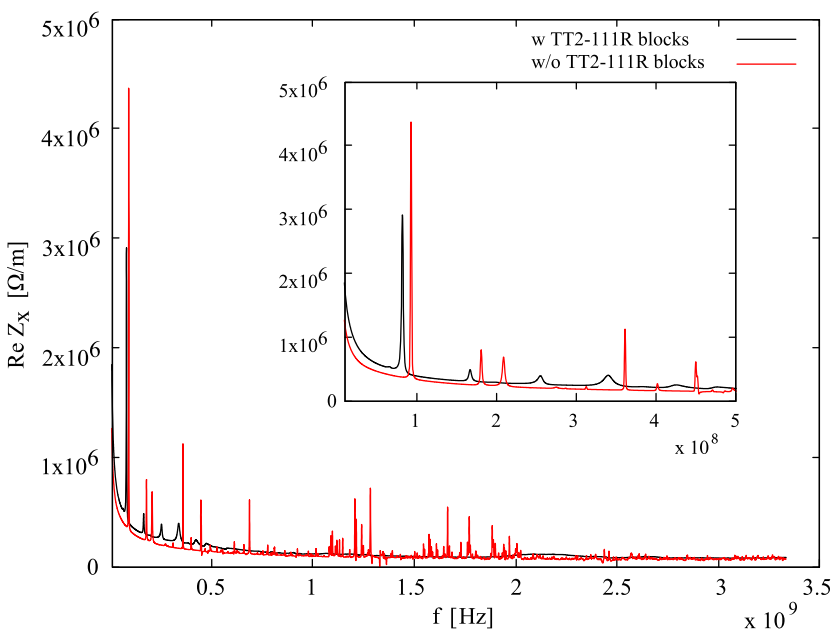

(a)

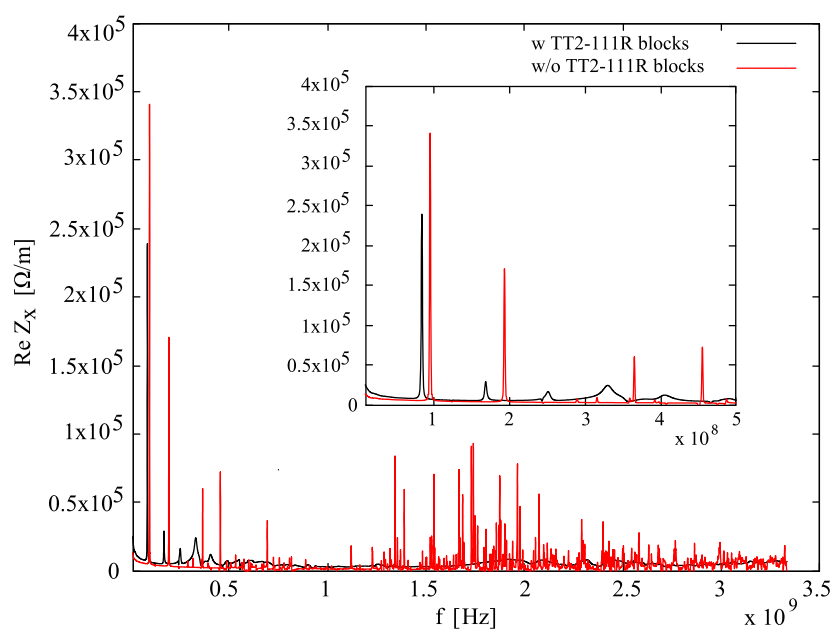

(b)

FIG. 6. Transverse dipolar impedance of the TCT real structure model calculated with GdfidL, for (a) $3 \mathrm{~mm}$ of half gap and (b) $8 \mathrm{~mm}$ half gap collimators, with tungsten jaws. The inset layers focus on the low frequency HOMs up to $500 \mathrm{MHz}$. The red curves correspond to the simulated structures without TT2-111R ferrite blocks, whereas the black ones to simulated structures with ferrite blocks. The HOMs characteristic frequencies are clearly shown to shift downward as an effect of the ferrite so as the HOMs shunt impedances being damped. frequency range between 1.2 and $1.4 \mathrm{GHz}$, located in the rf screen fingers, remain almost undamped. Besides, the resistive walls and the TT2-111R give some broad-band impedance contribution at low frequencies. As will be discussed later, the first HOM measurements with loops have revealed a presence of low frequency modes that are not seen in Fig. 4. In order to discriminate the longitudinal or transverse nature of the measured HOM, the longitudinal impedance was also calculated for a beam having a horizontal transverse offset. The results shown in Fig. 5 demonstrate that the low frequency modes appear only for the offset beam, thus corresponding to transverse modes. The frequencies obtained in simulations are in good agreement with those measured experimentally.

In order to define the parameters of these modes and to compare them with available transverse impedance measurements we have studied the transverse narrow band impedance in more detail. The transverse wakes and impedances of the LHC Run II TCS/TCT collimator structures were calculated by means of GdfidL for $3 \mathrm{~mm}$ and $8 \mathrm{~mm}$ jaws half gaps. In order to investigate the transverse HOM damping efficiency, the collimators with and without dispersive properties of TT2-111R, but together with resistive $\mathrm{W}$ jaws, were considered. The results of this simulation campaign are shown in Fig. 6(a) and Fig. 6(b).

Differently from the longitudinal case, the TT2-111R ferrite resulted to be very effective in damping the transverse parasitic modes for frequencies above $500 \mathrm{MHz}$. The modes at lower frequencies are less damped. The residual transverse $\mathrm{HOM}$ at frequencies around $100 \mathrm{MHz}$ and $200 \mathrm{MHz}$ have non-negligible shunt impedances, as it is seen in Fig. 6(a) and Fig. 6(b). The calculated parameters of these modes are summarized in Table II.

The effect of the TT2-111R ferrite consists in a reduction of the HOM shunt impedances and also in a shift of their frequencies toward lower values. As an example, for $8 \mathrm{~mm}$ half gap, the first HOM frequency shifts from about $95 \mathrm{MHz}$ down to $85 \mathrm{MHz}$, at exactly the same frequency measured experimentally with the loop techniques. The same is valid also for the second HOM frequency at

TABLE II. Summary of the first 2 HOM frequencies $f$ and transverse shunt impedances $R_{s}$ for $3 \mathrm{~mm}$ and $8 \mathrm{~mm}$ half gaps from GdfidL simulations.

\begin{tabular}{lccccc}
\hline \hline & \multicolumn{4}{c}{ HOMs } \\
\cline { 2 - 3 } $\begin{array}{l}\text { Half gap } \\
(\mathrm{mm})\end{array}$ & \multicolumn{2}{c}{$\mathrm{w} / \mathrm{TT} 2-111 \mathrm{R}$} & & $\mathrm{w} / \mathrm{o} \mathrm{TT} 2-111 \mathrm{R}$ \\
\cline { 2 - 3 } \cline { 5 - 6 } \cline { 5 - 6 } & $f[\mathrm{MHz}]$ & $R_{s}[\mathrm{M} \Omega / \mathrm{m}]$ & & $f[\mathrm{MHz}]$ & $R_{s}[\mathrm{M} \Omega / \mathrm{m}]$ \\
\hline & 82.6 & 2.913 & & 93.4 & 4.370 \\
& 167.2 & 0.485 & & 181.1 & 0.797 \\
& 84.7 & 0.239 & & 95.7 & 0.340 \\
\hline \hline
\end{tabular}


$169 \mathrm{MHz}$. The calculated shunt impedances of the two modes are in a reasonable agreement with the measurement results. For both $3 \mathrm{~mm}$ and $8 \mathrm{~mm}$ half gaps, the results agree within a factor of two, as it will be shown in Fig. 16 and will be discussed in the following section. For instance, for the case of $8 \mathrm{~mm}$ half gap the shunt impedance of the first $\mathrm{HOM}$ obtained numerically is $239 \mathrm{k} \Omega / \mathrm{m}$ to be compared with $152 \mathrm{k} \Omega / \mathrm{m}$ measured experimentally.

\section{IMPEDANCE MEASUREMENTS}

A campaign of measurements on one of the new TCT collimators was launched in 2014 at CERN. Figure 7 shows the collimator installation together with the rf measurements setup. Two types of measurements were performed: probe/loop measurements and stretched wire impedance measurements. The first allows for detecting possible low frequency local resonant modes within the structure, while the second gives a direct measurement of the longitudinal beam coupling impedance.

\section{A. Measurements with probe and loop}

In order to find presence of low frequency resonant modes, we used a $43 \mathrm{~cm}$ long probe. The termination part was modified in order to test different coupling mechanisms: we used a straight, a horizontally bent, and a loop termination as shown in Fig. 8. The probe ensures a good coupling to the electric field of possible resonant modes while the loop couples mainly to the magnetic field. Figure 9 shows the comparison of the three methods: looking at the S21 parameter we conclude that the probes cannot excite all the low frequency modes that are instead clearly visible using the loop excitation. The first three modes are located at the frequency of 87, 169, and $255 \mathrm{MHz}$. Looking at the S11 (and similarly for the S22), the large resonances at $\simeq 288 \mathrm{MHz}$ and $576 \mathrm{MHz}$ are the first two quarter-wave resonances characteristic of

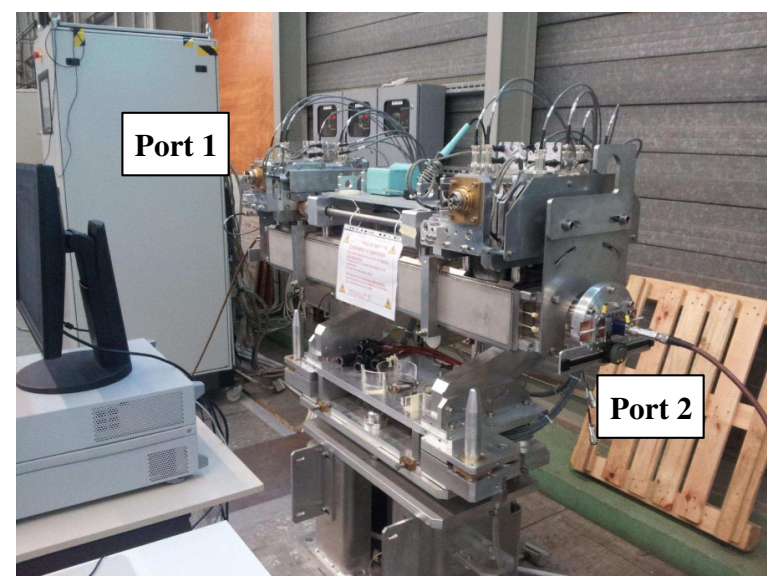

FIG. 7. The TCT collimator used during the rf impedance measurements campaign. Ports 1 and 2 are the VNA ports used for the reflection and transmission measurements.

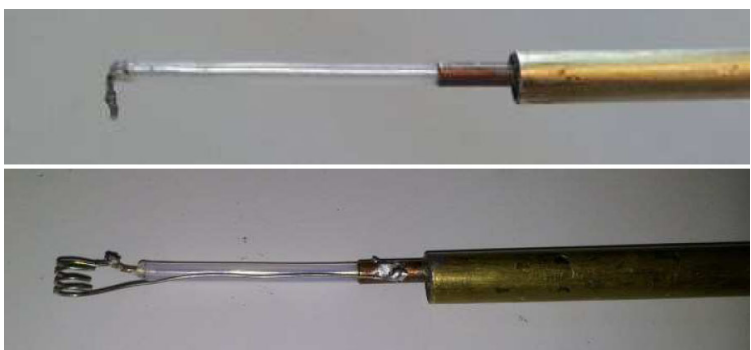

FIG. 8. Probe with bent termination (top) and probe with straight termination (bottom) on which has been mounted a loop.

the coaxial resonator made by the probes (which acts like the inner conductor) and the device (which acts like the outer conductor). These modes can be easily distinguished from the device resonant modes as their frequency shifts when moving the probe position. Alternatively, one could use absorbing foam at the inner side of the probe mounting plates to damp these additional modes.

The unloaded $Q$ for the first three low frequency modes was calculated in transmission ensuring low coupling of the loops for different jaw gap positions. This can be achieved retracting the probes at the two ports until a minimal coupling factor, usually less than a percent, is obtained (see for example [27] on the use of the Smith chart to achieve this). The result can be seen in Fig. 10 and shows very little dependence of the unloaded $Q$ versus the collimator gap.

\section{B. Stretched wire setup}

In order to measure the device impedance, the stretched wire method is applied. The stretched wire method is based on the idea that the field excitation induced by a current pulse traveling on a wire stretched along a beam pipe, is similar to the one induced by a traveling bunch of charged
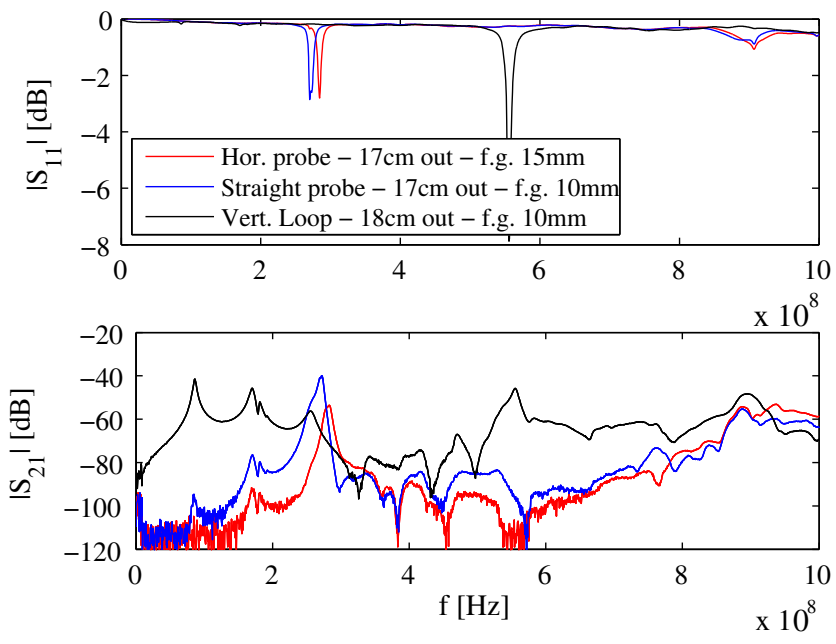

FIG. 9. S11 (top) and S12 (bottom) measured with a straight and horizontal probe and with a loop: the low frequency resonant modes exhibit higher coupling through the magnetic field. The first length in the legend refers to the portion of probe outside the device, the second ("f.g.") to the full gap of the collimator. 


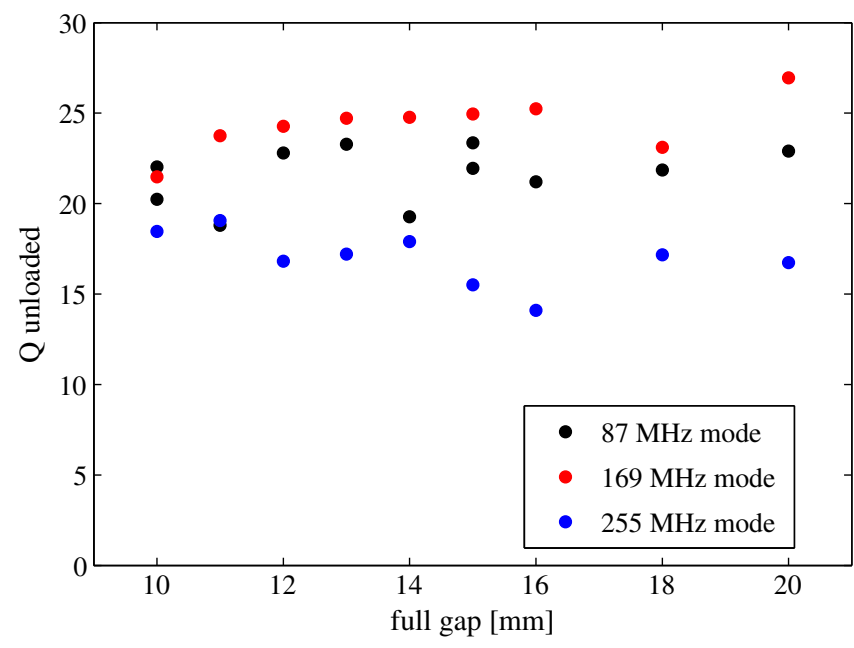

FIG. 10. Dependence of the unloaded $Q$ versus jaw full gaps for the first three low frequency resonant modes.

particles [28]. Inserting a thin wire in a vacuum chamber, we transform it in a coaxial transmission line. The transmission scattering coefficient S21 of the line can be therefore measured using a vector network analyzer (VNA) and provides an observable for those fields having an electrical longitudinal component at the center of the beam pipe. This method is commonly adopted to measure the longitudinal beam coupling impedance of a device [29].

To perform the measurements, the coaxial line made by the wire and the beam pipe has to be adapted to the $50 \Omega$ impedance of the VNA cables. This can be done using a matching network. As a simple and practical solution, we considered a single resistor $Z_{m}$ in series to the characteristic impedance $Z_{c}$ of the coaxial line before and after the DUT, as shown in Fig. 11. In order to match the $50 \Omega$ cables we need to set $Z_{m}=Z_{c}-50 \Omega$. We stress the fact that, in reality, the matching is not perfect due to the slightly frequency dependent value of the matching resistors.

The use of additional attenuators mitigates the mismatch between the cables and the VNA which is only partially handled by the calibration.

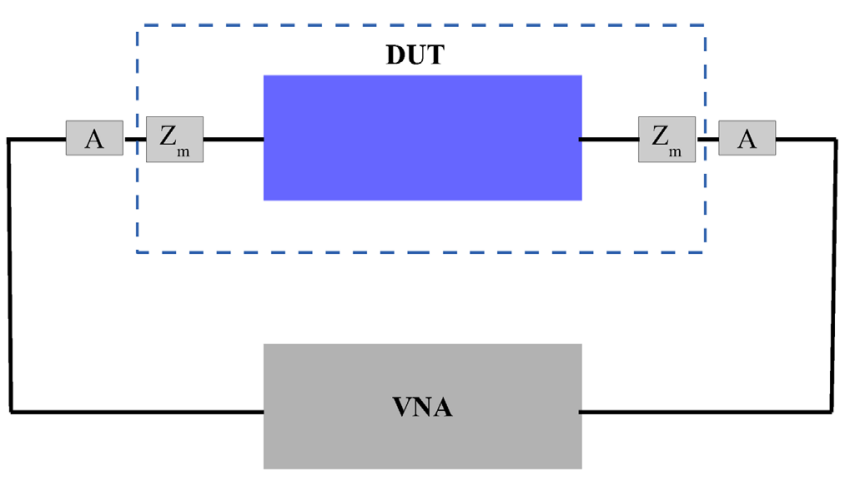

FIG. 11. Stretched wire setup: a single wire is stretched along the device under test (DUT). The impedance matching is ensured by the network (block " $Z_{m}$ ") and the series attenuator (block "A").
The coaxial transmission line impedance made by the collimator and the wire can be directly measured through the time domain reflectometry (TDR). Measuring the S11 and S22 from the two ports and transforming to the impedance seen in reflection for example on port 1 we define $Z_{11}(f)$ as

$$
Z_{11}(f)=Z_{0} \frac{1+S_{11}(f)}{1-S_{11}(f)},
$$

with $Z_{0}$ the cable $50 \Omega$ line impedance. Performing an inverse Fourier transform $\mathcal{F}^{-1}$ we have

$$
Z_{11}(t)=\mathcal{F}^{-1} Z_{11}(f),
$$

that corresponds to the impulse response of the DUT in reflection from port 1 . Convoluting with a step function we get the DUT step response: this quantity is particularly useful in order to identify the impedance change along a coaxial transmission line.

Figure 12 shows the characteristic impedance for different positions of the jaws gap as a function of the collimator length: for large gaps the impedance is close to $300 \Omega$ with small deviations towards the start and the end of the collimator due to the double BPM taperings and longitudinal rf fingers; decreasing the gap, the collimator impedance decreases mainly along the jaw position up to $100 \Omega$ for $10 \mathrm{~mm}$ of full gap. In order to properly match the transmission line to the $50 \Omega$ VNA cable we should in principle match each gap with its corresponding matching resistor: this is not practicable due to the time needed for soldering the resistors between the VNA cables and the wire. Moreover, moving the jaws we would not be able to match both the double BPM tapering and the collimator

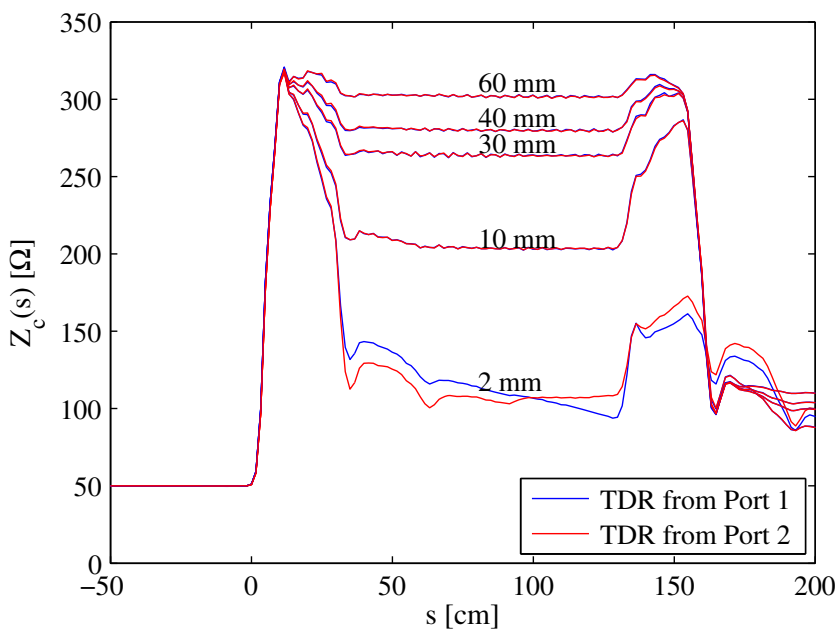

FIG. 12. TDR measurements from the two ports of the collimator for different values of the full jaws gap. The double BPM tapering and longitudinal rf finger transitions to the jaws are visible. Moving the jaws toward smaller values of full gap the characteristic transmission line impedance $Z_{c}$ is reduced. 
jaws. An intermediate value has been therefore chosen as $Z_{c}=230 \Omega$ from which we deduce $Z_{m}=180 \Omega$. As a consequence we can already expect to have a considerable mismatch when measuring the impedance: this will mainly affect the broadband part of the impedance while possible resonant modes can still be detected and measured.

\section{Stretched wire measurements}

Measurements were performed displacing the wire in steps of $0.25 \mathrm{~mm}$ for three values of full gap: 5, 10, and $15 \mathrm{~mm}$. Figure 13 shows the typical S21 signal measured with the VNA: the standing wave pattern is introduced by the impedance mismatch at the BPM and transition locations. Nevertheless, we can appreciate some discontinuities in the pattern around $\simeq 82$ and $\simeq 170 \mathrm{MHz}$ that are induced by the presence of the resonant modes measured with the probe at 87 and $169 \mathrm{MHz}$. The frequency shift is expected as it is due to the removal of the energy introduced by the wire presence. The baseline standing wave curve can be fitted and removed locally in order to isolate the resonant mode. Performing the baseline removal for each value of the displaced wire, we can get the S21 for each of the two detected resonant modes.

The device length is $L=1.2 \mathrm{~m}$ and the wavelength at the highest resonant mode frequency is $\lambda=1.5 \mathrm{~m}$. Being $L<\lambda$ and the expected longitudinal impedance $Z_{l}<Z_{c}$, we can use the log-formula to calculate the longitudinal impedance at each position of the wire [30]. Considering then, the wire displacement $x_{0}$ we derive the transverse impedance as [31]:

$$
Z_{x, \text { tot }}=\frac{c}{\omega} \frac{Z_{l}}{x_{0}^{2}}
$$

where $c$ is the speed of light and $\omega=2 \pi f$.

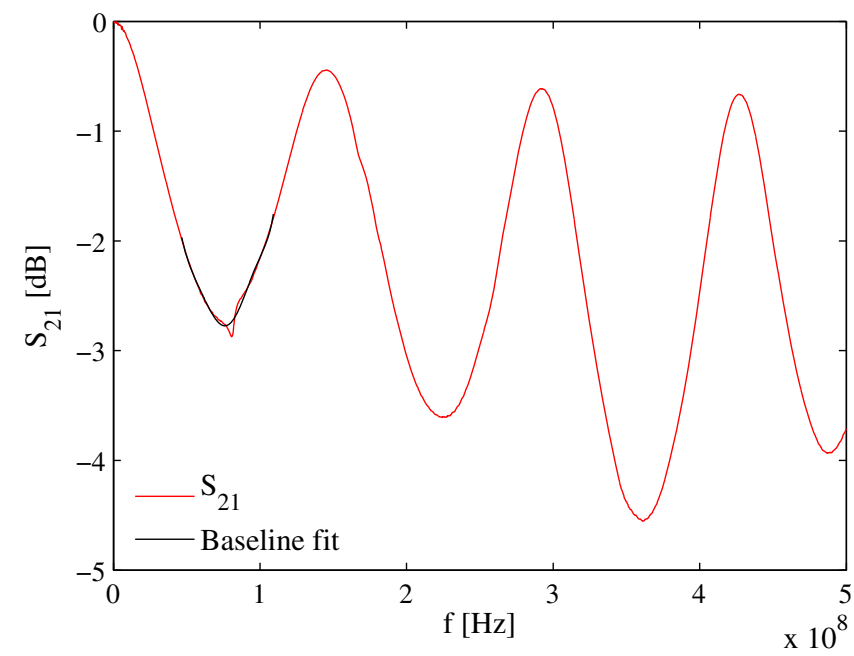

FIG. 13. Measured $S 21$ and baseline fit with the wire displaced by $5 \mathrm{~mm}$ and collimator jaws full gap of $10 \mathrm{~mm}$. Note that the offset induced by the matching resistor and the attenuators has been removed from the $S 21$.
The longitudinal impedance $Z_{l}$ for the first and second resonant modes is calculated for each gap and each wire position $x_{0}$. Figure 14 shows the peak values of $Z_{l}$ at the resonant mode frequencies as a function of the wire position. The parabolic behavior reveals that we are dealing with transverse modes [32]. We notice that the curves are not centered as a consequence of the asymmetric ferrite position.

Performing a parabolic fit we can determine the $Z_{x, \text { tot }}$ with the corresponding uncertainty. The result is shown in Fig. 15. According to the generalized theory of impedance for flat symmetric structures [32], $Z_{x, \text { tot }}=Z_{x, \text { dip }}-Z_{x, \text { quad }}$. This is not strictly true in our case as the ferrite is placed in asymmetric locations, but we can consider it as a first approximation.

A remarkably good agreement, within a factor of 2 , is obtained with GdfidL and CST simulations, as shown in Fig. 16

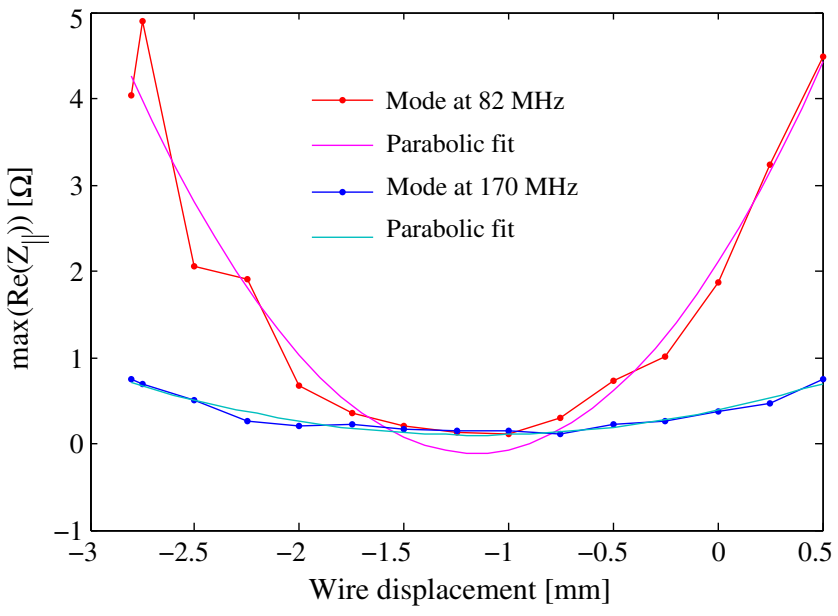

FIG. 14. Longitudinal impedance as a function of the wire displacement with respect to the geometrical center of the collimator aperture. The longitudinal impedance is calculated at the resonant frequency of the resonant modes.
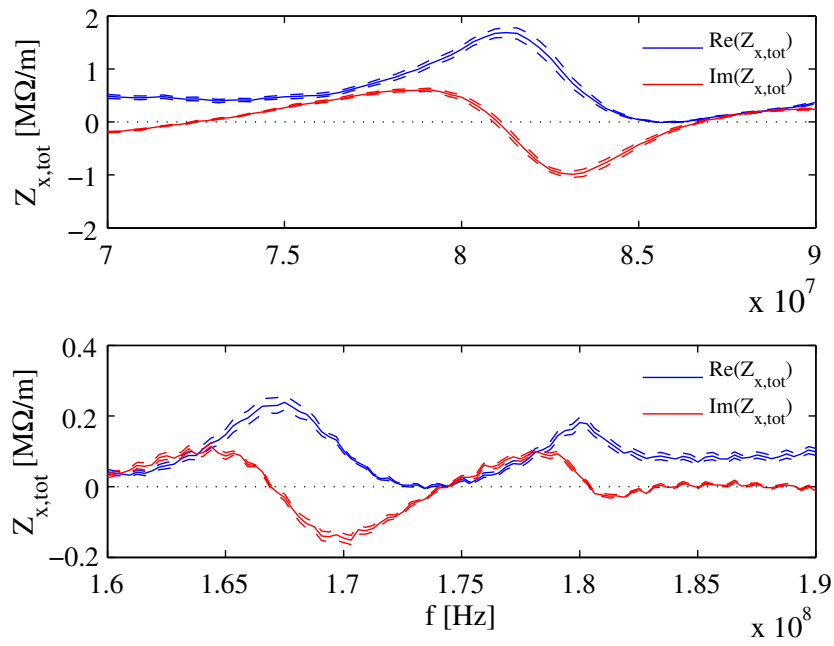

FIG. 15. Transverse impedance calculated at the full gap of $5 \mathrm{~mm}$. The dashed lines refer to the uncertainty of the measurement. 


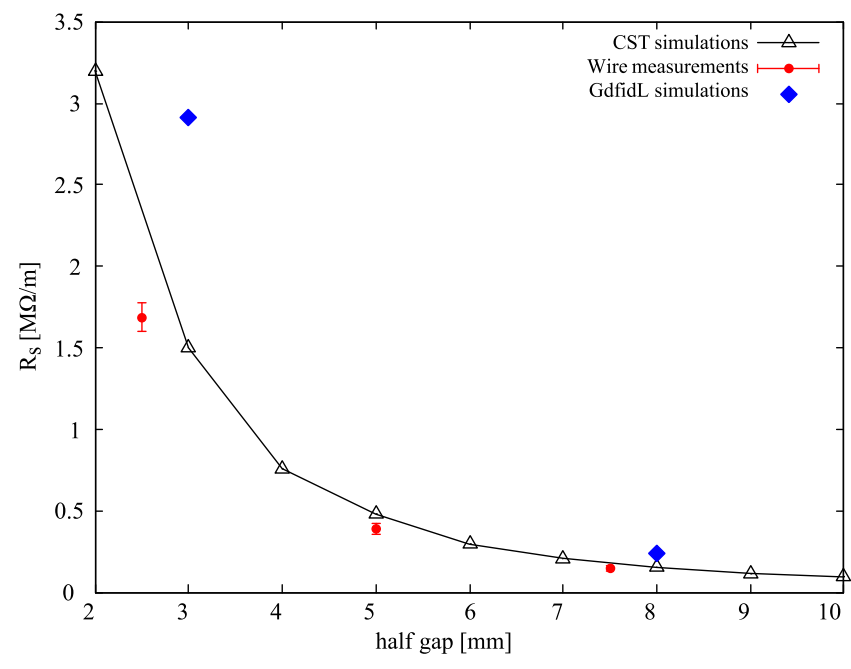

FIG. 16. Shunt impedance $R_{s}$ of the $87 \mathrm{MHz}$ mode versus half gap as measured with wire and simulated in CST and GdfidL.

for the $87 \mathrm{MHz}$ mode. The discrepancy may depend on several collimator design constraints, namely the gap between the plate where ferrite blocks are installed, the gap between collimator jaws and external tank and, also, on the mesh and computed wake length [20].

\section{HOM EFFECT ON BEAM STABILITY}

In order to estimate the effect of the low frequency HOMs, DELPHI simulations were performed [33,34] both in single and coupled bunch regime at $Q^{\prime}=5$ (where $Q^{\prime}=Q \xi$ with $Q$ machine tune and $\xi$ chromaticity in the plane of reference) and damper of 50 turns damping time. Figure 17 shows the effect of an additional HOM of variable resonant frequency $f, Q=20$, and $R_{s} \in(1,10,100) \mathrm{M} \Omega / \mathrm{m}$

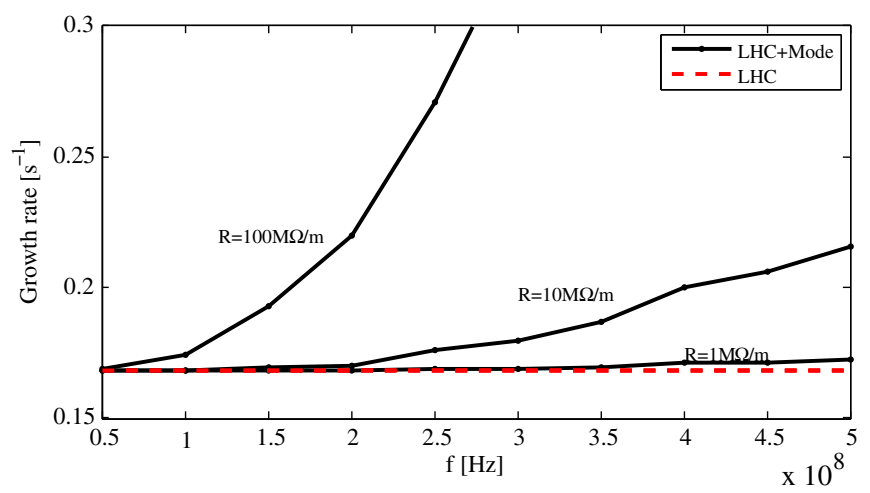

FIG. 17. Most unstable mode growth rate obtained adding on top of the 2016 LHC baseline impedance model an HOM with $Q=20$, variable frequency $f$ and shunt impedance $R_{s}$. The calculation is done for 2748 nominal LHC bunches of $N_{b}=1.2 \times 10^{11} \mathrm{ppb}$ intensity and $\sigma_{z}^{r m s}=9 \mathrm{~cm}$ bunch length at $Q^{\prime}=5$ with transverse damper gain of 50 turns. The total number of 8 TCTs is included in the simulations weighting for the betatron function at their location. on the transverse LHC growth rate of the most unstable mode among 2748 coupled bunch modes: being in the range of $1 \mathrm{M} \Omega / \mathrm{m}$, the HOMs introduced by the TCTs are not expected to be an issue for the LHC beam stability. Similar conclusions hold for the single bunch case.

\section{CONCLUSIONS}

The design upgrade of the TCS/TCT collimators in the LHC in order to host embedded button BPMs, represents an important step in machine cleaning and performance optimization. Nevertheless, this progress implies a compromise in terms of impedance as the rf contacts had to be removed introducing HOMs at lower frequencies, more dangerous as deeper inside the LHC beam spectrum. In the frame of this work, we carefully quantified the impact of the design upgrade in terms of impedance with both simulations and experimental measurements and demonstrated the negligible effect on the LHC machine stability. The HOMs introduced with the device upgrade, have been effectively mitigated by means of ferrite blocks. The undamped modes below $500 \mathrm{MHz}$ have been identified with both GdfidL and CST simulations and experimentally confirmed with rf bench impedance measurements. These modes are predicted not to be an issue for the LHC and, after installation in the machine, no issues have been recorded yet.

[1] R. W. Assmann et al., in Proceedings of the 8th European Particle Accelerator Conference, Paris, 2002 (EPS-IGA and CERN, Geneva, 2002), p. 197.

[2] R.W. Assmann, in Proceedings of the 46th ICFA Advanced Beam Dynamics Workshop on High-Intensity and High-Brightness Hadron Beams HB2010, Morschach, Switzerland, 2010 (PSI, Villigen, Switzerland, 2010), p. 21.

[3] A. Bertarelli et al., in Proceedings of the 9th European Particle Accelerator Conference, Lucerne, 2004 (EPS-AG, Lucerne, 2004), p. 545 [http://accelconf.web.cern.ch/ AccelConf/e04/].

[4] N. Mounet, Ph.D. thesis, Ecole Polytechnique, Lausanne, Switzerland, 2012.

[5] E. Métral et al., in Proceedings of the 22nd Particle Accelerator Conference, PAC-2007, Albuquerque, NM (IEEE, New York, 2007), p. 2003.

[6] N. Mounet et al., in LHC Beam Operation Workshop, Evian, France, 2012 (CERN, Geneva, Switzerland, 2013).

[7] B. Salvant et al., in LHC Performance Workshop, Chamonix, France, 2014 (CERN, Geneva, Switzerland, 2015).

[8] E. Métral, Beam intensity limitations, Deliverable: D2.4, Technical report, CERN, Geneva, Switzerland, 2014.

[9] N. Biancacci, K. Li, E. Métral, and B. Salvant, in Proceedings, 7th International Particle Accelerator Conference (IPAC 2016), Busan, Korea, 2016 (JACoW, Geneva, Switzerland, 2016). 
[10] D. Wollmann et al., Beam feasibility study of a collimator with in-jaw beam position monitors, Nucl. Instrum. Methods Phys. Res., Sect. A 768, 62 (2014).

[11] G. Valentino, A. A. Nosych, R. Bruce, M. Gasior, D. Mirarchi, S. Redaelli, B. Salvachua, and D. Wollmann, Successive approximation algorithm for beam-positionmonitor-based LHC collimator alignment, Phys. Rev. Accel. Beams 17, 021005 (2014).

[12] S. DeBarger et al., Report No. SLAC-PUB-11752.

[13] A. Novokhatski, F.-J. Decker, H. Smith, and M. Sullivan, Wakefields in SLAC linac collimators, Phys. Rev. Accel. Beams 17, 124401 (2014).

[14] Y. Suetsugu, K. Shibata, A. Morishige, Y. Suzuki, and M. Tsuchiya, Design study of a movable mask with low beam impedance, Phys. Rev. Accel. Beams 9, 103501 (2006).

[15] Y. Suetsugu, K. Shibata, T. Ishibashi, K. Kanazawa, M. Shirai, S. Terui, and H. Hisamatsu, First commissioning of the SuperKEKB vacuum system, Phys. Rev. Accel. Beams 19, 121001 (2016).

[16] M. Zobov et al., Report No. Preprint LNF-95-041-P, 1995.

[17] A. Dallocchio, A. Bertarelli, C. Boccard, F. Carra, M. Gasior, L. Gentini, and M. A. Timmins, in Proceedings of the 2nd International Particle Accelerator Conference, San Sebastián, Spain (EPS-AG, Spain, 2011).

[18] E. Métral et al., in Proceedings of the 4th International Particle Accelerator Conference, IPAC-2013, Shanghai, China, 2013 (JACoW, Shanghai, China, 2013).

[19] H. A. Day, Ph.D. thesis, University of Manchester, 2012.

[20] O. Frasciello, Ph.D. thesis, University of Rome "La Sapienza," 2015.

[21] W. Bruns, http://www.gdfidl.de.
[22] CST Studio Suite ${ }^{\circledR}$, http://www.cst.com.

[23] TT2-111 data sheet available at http://www.trans-techinc .com/products_detail.asp?ID $=151$.

[24] O. Frasciello and M. Zobov, in Proceeding of 12th International Computational Accelerator Physics Conference (ICAP2015), Shanghai, China, 2015 (JACoW, Geneva, Switzerland, 2016).

[25] N. Biancacci, V. G. Vaccaro, E. Métral, B. Salvant, M. Migliorati, and L. Palumbo, Impedance studies of 2D azimuthally symmetric devices of finite length, Phys. Rev. Accel. Beams 17, 021001 (2014).

[26] O. Frasciello, S. Tomassini, M. Zobov, B. Salvant, A. Grudiev, and N. Mounet, Geometric beam coupling impedance of LHC secondary collimators, Nucl. Instrum. Methods Phys. Res., Sect. A 810, 68 (2016).

[27] F. Caspers, RF engineering basic concepts: The Smith chart, arXiv:1201.4068.

[28] M. Sands and J. Rees, SLAC-Report No. PEP-95, 1974.

[29] V. G. Vaccaro, Coupling Impedance Measurements: An Improved Wire Method (INFN, Naples, 1994).

[30] A. Mostacci and F. Caspers, in ICFA mini-Workshop on "Electromagnetic wake fields and impedances in particle accelerators," Erice, Italy, 2014.

[31] G Nassibian and F. J. Sacherer, Methods for measuring transverse coupling impedances in circular accelerators, Nucl. Instrum. Methods 159, 21 (1979).

[32] E. Métral and G. Rumolo, USPAS course, Albuquerque, New Mexico, USA, 2009.

[33] N. Mounet, in HSC meeting, CERN, Geneva, Switzerland, 2014.

[34] N. Mounet, in Impedance meeting, CERN, Geneva, Switzerland, 2013. 